\title{
Fabrication of multimode tip fiber sensor based on surface plasmon resonance (SPR)
}

\author{
Nidaa L. Al- Janaby ${ }^{1 *}$, Anwaar A. Al-Dergazly ${ }^{2}$ \\ ${ }^{12}$ Laser and Optoelectronics Engineering Department, Collage of Engineering, Al-Nahrain University, Iraq
}

*Corresponding author: nidaalafta@ yahoo.com

\section{(c) The Author}

2020.

Published by

ARDA.

\begin{abstract}
In this research, a fiber optic sensor based on surface plasmon resonance (SPR) was prepared. The sensor of SPR was configured using a gold thin layer at the end of a cleaved optical fiber by a sputtering technique. The source of white light was utilized to produce a series of wavelengths and excites surface plasmon resonance at the fiber tip. The SPR sensor was immersed into media with different refractive indices in the range of 1 to 1.58 , including their similar plasmon resonance wavelength shifts that were saved by the optical spectrum analyzer. Experimental results that were obtained show there is a redshift when increasing the refractive index of solutions and sensitivity reach $298 \mathrm{~nm} / \mathrm{RIU}$, and resolution $4.31 \times \llbracket 10 \rrbracket^{\wedge}(-4)$.
\end{abstract}

Keywords: Tip optical fiber sensor, Surface plasmon resonance sensor (SPR),

Reflective sensor; Refractive index sensor

\section{Introduction}

Surface plasmon resonance (SPR) is a physical phenomenon that occurs when electrons oscillation at the interface between a thin metallic film (gold or silver) and a dielectric medium (glass, liquid, gas) [1]. SPR sensing has been investigated intensively because of its unique advantages such as large accuracy, active analysis, and no need for labeling [2]. Conventionally, the SPR sensor is measured using the Kretschman structure as shown in Figure1 including the prism and a thin metal coating (e.g. silver or gold) covered on bottom of prism for reflecting. Both silver and gold are utilized to excite SPR in the visible and near infrared range [3].



Figure 1. Kretschmann SPR configuration

This work is licensed under a Creative Commons Attribution License (https://creativecommons.org/licenses/by/4.0/ ) that allows others to share and adapt the material for any purpose (even commercially), in any medium with an acknowledgement of the work's authorship and initial publication in this journal. 
For produce total internal reflection (TIR) which sensitive into outside sensing medium, utilizing gold (Au) as the plasmonic material coating directly above the base of prism [4]. A minute change with a refractive index regarding the sensing medium creates an increase to the similar variation with propagation constant of SPR and a high shift of spectral within resonance dips. The refractive index variation estimated by recognizing the spectral shift of resonant dips. Depend on the same system in Kretschmann prism that associated, the SPR with fiber optic technology owns great improvements with giving advantages exceeding the common prism based SPR reaches, such as a low cost, miniaturization, and simplicity [5]. SPR fiber sensors can operate in the reflection mode (fiber tip), a sensor consisting of the fiber with a perfect cleave and a layer of gold or another plasmonic material at one end of the fiber, which reflects light towards the detector at the opposite end [6].

\section{Plasmons theory}

The resonance phenomenon created surface plasmon waves (SPWs) is stimulated by the metal-dielectric interface. The electric field (E) of the (SPWs) propagating in the $x$-direction is represented by equation (1) and shown in Figure 1 [7]:

$E=E_{o} \exp \left[i\left(k_{x} x \pm k_{z} z-\omega t\right)\right] \quad \ldots(1)$

The plus and minus signs are for $z>=0$ and $z<=0$ respectively. The propagation constant $k_{z}$ is imaginary, which causes the exponential decay of the field. The wave vector $K_{x}$ is parallel to the $x$-axis and is given as equation (2) [8]:

$k_{x}=\frac{2 \pi}{\lambda_{p}}$

$\lambda_{p}$ is plasma wavelength.

The field associated with SPWs excitations has exponentially decays in metal and dielectric media, as shown in Figure 2, where SPW is excited [9].

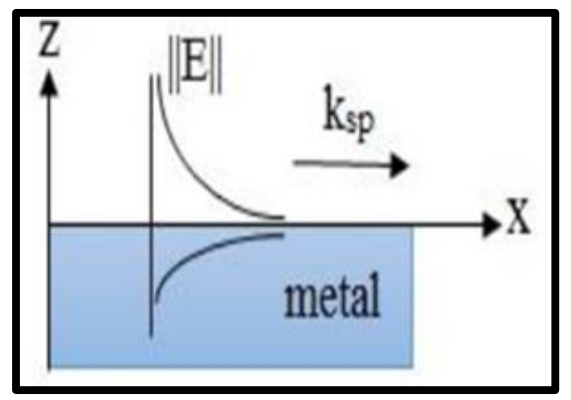

Figure 2. The exponential decay of the electromagnetic fields on every side of the interface

The propagation constant of SPW depends on the dielectric constant of the outer medium in connection with the metal as shown as equation (3):

$$
k_{s p}=\frac{\omega}{c} \sqrt{\frac{\varepsilon_{m} \varepsilon_{d}}{\varepsilon_{m}+\varepsilon_{d}}}
$$

where:

$\varepsilon_{m}$ and $\varepsilon_{d}$ are the dielectric constants of metal and dielectric respectively, $\omega$ is the frequency of incident light and $c$ is the speed of light in vacuum. 
It is important to provide the equality between the wave vector of the surface plasmons $k_{s p}$, and the wave vector $k_{x}$ of the incident light to achieve resonance condition. This can be written easily according to equation (4) $[10]$ :

$$
K_{x}(\text { photon })=K_{s p}
$$

This coupling condition means that resonance occurs and leads to carry energy from incident light to surface Plasmon wave, the outside medium connects with the metal. By employing the boundary conditions on the dielectric metal interface, the dispersion relation concerning surface plasmon wave can be achieved to be:

$k_{s p}=k_{x}=\frac{\omega}{c} \sqrt{\frac{\varepsilon_{m} \varepsilon_{d}}{\varepsilon_{m}+\varepsilon_{d}}}$

There are two forms of the SPR methods detection according to the resonance coupling condition given by equation (6) [11]:

$\frac{\omega}{c} \sqrt{\varepsilon_{p}} \sin \theta=\frac{\omega}{c} \sqrt{\frac{\varepsilon_{m} \varepsilon_{d}}{\varepsilon_{m}+\varepsilon_{d}}}$

where, $\varepsilon_{p}$ is dielectric function of the prism, $\theta$ is incident angle.

The first form is angular interrogation of SPR that measure the variation in resonance angles, at a fixed wavelength $\lambda$. The second form is known as spectral SPR, which measures the variation in resonant wavelengths at a fixed angle of incidence $\theta$. The difference between angular SPR and spectral SPR is that spectral SPR needs to utilize costly and complicated tools. However, the feature of spectral SPR has the potential to utilize fiber optic for remote sensing.

\subsection{Performance characteristics}

The benefit and limitations of the sensor are determined according to the performance parameters such as sensitivity $(S)$, signal to noise ratio (SNR), figure of merit (FOM) and resolution (R) [12]. In the case of spectral (wavelength) interrogation, sensitivity $(S)$, can be determined as follows per Equation (7):

$$
S=\frac{\Delta \lambda_{\text {res }}}{\Delta n_{s}}
$$

SNR and FOM are inversely proportional to the width of the SPR spectral curve and can be formulated per Equations $(8,9)$ :

$$
\begin{aligned}
& S N R=\left[\frac{\Delta \lambda_{\text {res }}}{\Delta \lambda_{0.5}}\right] \\
& \text { FOM }=\frac{\mathrm{S}}{\Delta \lambda_{0.5}}
\end{aligned}
$$

Lastly, the resolution of the sensor can be described as the smallest of variation in refractive index detectable by the sensor and presented as equation (10) [12]:

$$
R=\frac{\Delta n_{s}}{\Delta \lambda_{\text {res }}} \Delta \lambda_{D R}
$$

where, $\Delta \lambda_{D R}$ is the spectral resolution of the spectrometer. 


\section{Experimental arrangement}

\subsection{Preparation of optical fiber SPR sensor probe}

The procedure of fabrication plasmonic fiber optic sensor involves two steps. The first one includes preparation of fiber for deposition plasmonic material. It starts with using a piece of $10 \mathrm{~cm}$ multimode optical fiber with core diameter of $50 \mu \mathrm{m}$ and cladding diameter of $125 \mu \mathrm{m}$. Then, removing the protective coating along the optical fiber, which consists of three-layers (outer jacket, buffer coating, and acrylate coating) by a stripper and lastly, cleaving the end of the fiber to get a flat surface in order to provide fiber tip for gold deposition. The second step includes gold deposition on the tip of fiber to produce a reflective sensor using physical vapor deposition (PVD), which is known as the sputtering technique. In this technique, the thickness of the gold film depends on twoparameters: the coating current and the ion sputtering deposition time. Increased coating time leads to increase thickness of gold film. For the purpose of this research, different coating times were used starting from 30s, 60s, 120s, 180s, 300s. Atomic force microscopy (AFM) showed that the optimum coating time is $60 \mathrm{~s}$. Gold particle roughness with its size and diameter were examined by AFM and shown in Figure 3a and Figure 3b. It was shown that the average grain size as determined from the atomic force microscope is $100.22 \mathrm{~nm}$ and average surface roughness is $3.87 \mathrm{~nm}$. The average grain size of $10 \%$ from the gold film is about $50 \mathrm{~nm}$ while the average grain size of $90 \%$ from the gold film is about $150 \mathrm{~nm}$. This will be the effect on the wavelength resonance shift.
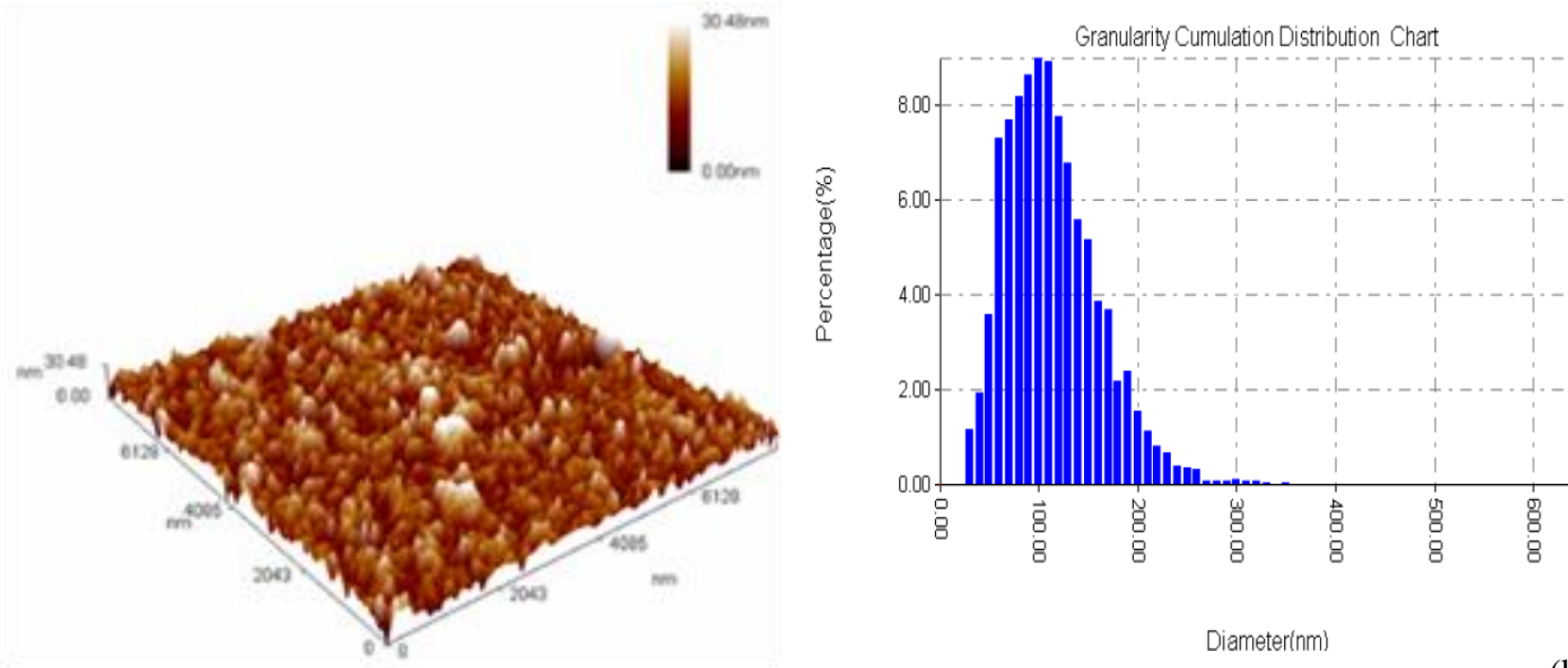

(a)

Diameter(nm)

Figure 3. (a) 3D-AFM image of fiber; (b) AFM Granularity accumulation of immobilized $A_{u}$ nanoparticles

The morphology concerning the prepared SPR optical fiber sensor was investigated via SEM as given in Figure 4.

Metalized fiber connected with a 1x2 mm optical coupler with a core diameter of $62.5 \mu \mathrm{m}$ and cladding diameter $125 \mu \mathrm{m}$. The variation in the core diameters between the reflective MMF sensor and the fiber coupler makes the light leakage into the cladding of the MMF sensor.

When the leaked light is reflected at the edge of the cladding region and the surrounding environment according to conditions of total internal reflection (TIR). The light wave excites the evanescent wave on the 
MMF sensor. Following the fabrication step, the work goes to the examination sensor. The spectral return measured concerning the RI variation in the surrounding medium. Results with different refractive index to examination sensor are shown in Table 1.

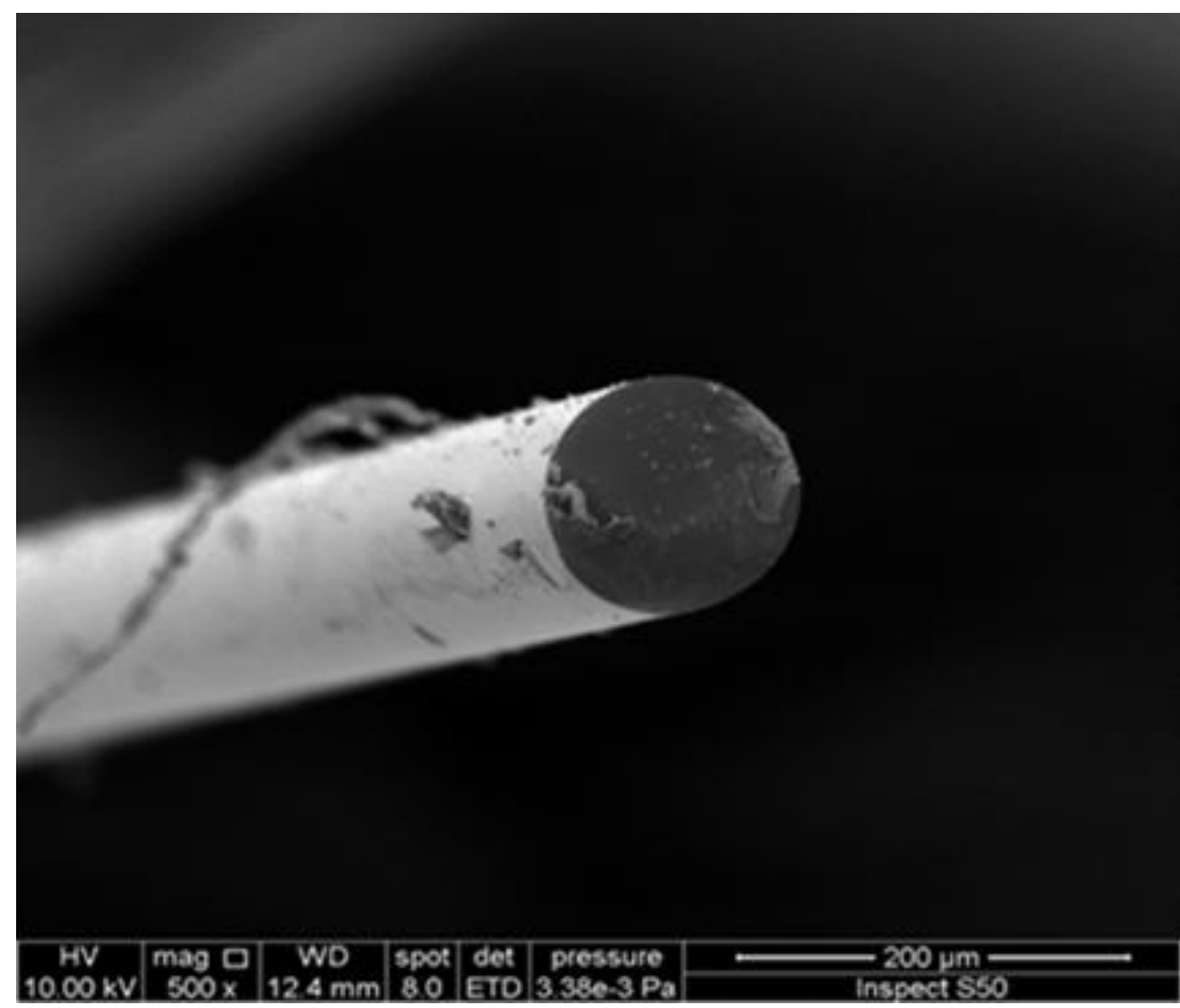

Figure 4. SEM image of immobilized Au coating on the tip of the fiber

Table 1. Refractive index results

\begin{tabular}{cc}
\hline Medium & Refractive index \\
\hline Air & 1 \\
Distill Water & 1.33 \\
Ethanol & 1.36 \\
Aniline & 1.582 \\
\hline
\end{tabular}

\subsection{Experimental setup}

The measurement system of the optical fiber SPR sensor as a schematic description is shown in Figure 5a. The system consists of a halogen light source, a1x2 fiber coupler (Y-form), an optical spectrum analyzer (OSA), a computer provided with data processing software, a reflective SPR fiber sensor head and measuring cell. White light emitted from the halogen light source that connected to a $1 \times 2$ fiber coupler and transmitted to the sensing zone within one port of the fiber coupler. Which enters the OSA (Thorlabs-CCS200), through another port of the fiber coupler.

According to resonance condition if the wave vector of the incident light equals the wave vector of surface plasmon SPR wave can be resonantly excited. Finally, the variation in the refractive index can be received from the output spectrum exhibited on the computer, as shown in the experimental setup of Figure $5 \mathrm{~b}$. 


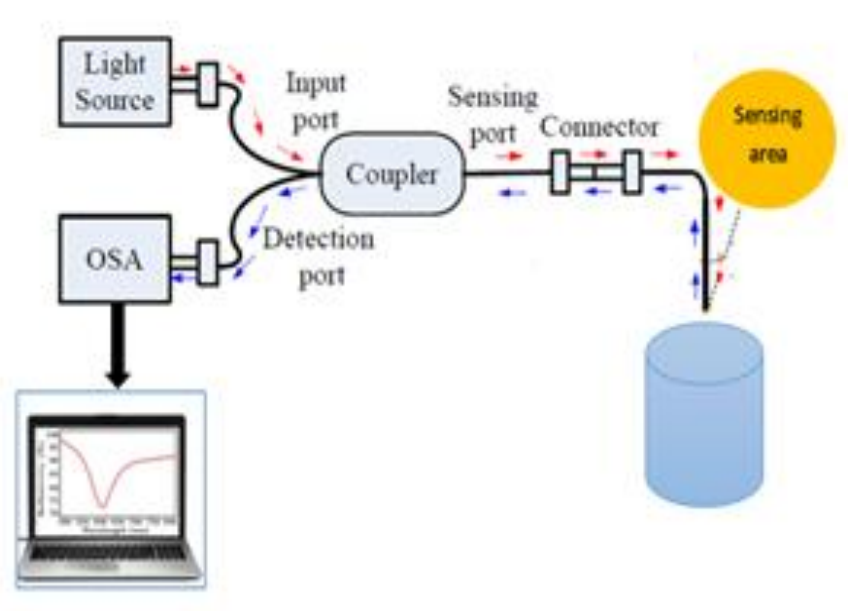

(a)

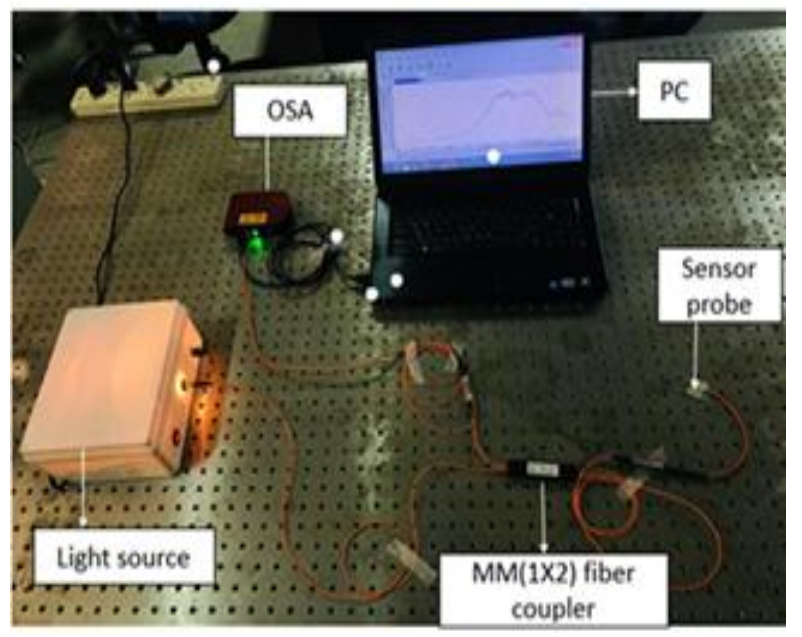

(b)

Figure 5. (a) The schematic experimental setup system; (b) image of an experimental setup SPR optical fiber sensor

\section{Results and discussion}

The ability concerning the SPR fiber optic sensor to discover the variation of the surrounding refractive index (RI) within the noticeable spectrum is first tested. For measuring the spectral development, halogen lamp and optical spectrum analyzer were applied, and different media including various RIs arranging from 1 to 1.58 put inside the measuring cell. The software equipped with OSA record the output spectrum, $I_{0}$ that represents origin spectrum of the polychromatic light without sensor (bare sample) measured using the spectrum analyzer; that represent with black line then insert the sensing part into light source and record the output spectrum $I_{1}$ in air (after coating) that represents with red line as shown in Figure 6a. Finally, measure of the response spectrum by subtraction $I_{1}$ from $I_{0}$ to receive reflectance, which was calculated directly from the spectrum in Figure 6a that represent dip of resonance wavelength as shown in Figure $6 \mathrm{~b}$.

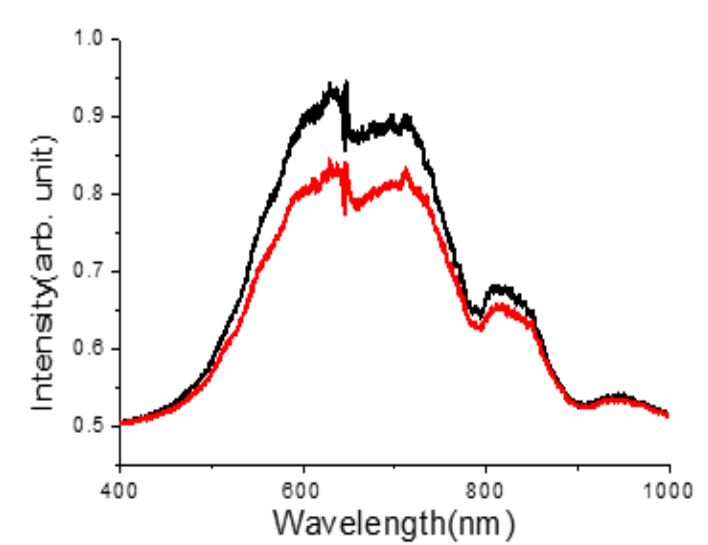

(a)



(b)

Figure 6. (a) The spectrum of the polychromatic light through measured origin spectrum for a bare region of optical fiber, sensitive region in the air with $\mathrm{n}=1$; (b) Reflected power

The same procedure with a separate solution to sensitive variation in refractive index and notice the shift with wavelength was made. Observation cleared plasmonic resonant wavelength redshift when increasing the refractive index of solvent. Therefore, resonant wavelength shifts from $709.12 \mathrm{~nm}$ to $800.41 \mathrm{~nm}$, can be seen that there is a change $(91.28 \mathrm{~nm})$ in the dip of wavelength position when the sensor was changed from air to 
aniline, when the index refraction of the solvent rises from (1 to 1.58) as shown in Figure7a. With other media (water, ethanol, and aniline; refractive index 1, 1.33, 1.36, 1.58), sensitive variation in the refractive index appears position of the dip is moved to the right (longer wavelength) when the external refractive index of the solution medium is increased as shown in Figure $7 \mathrm{~b}$.

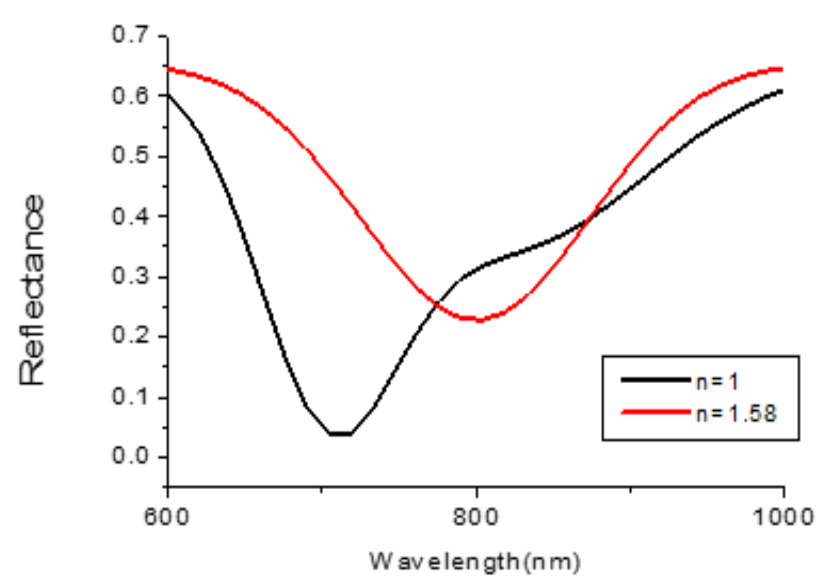

(a)

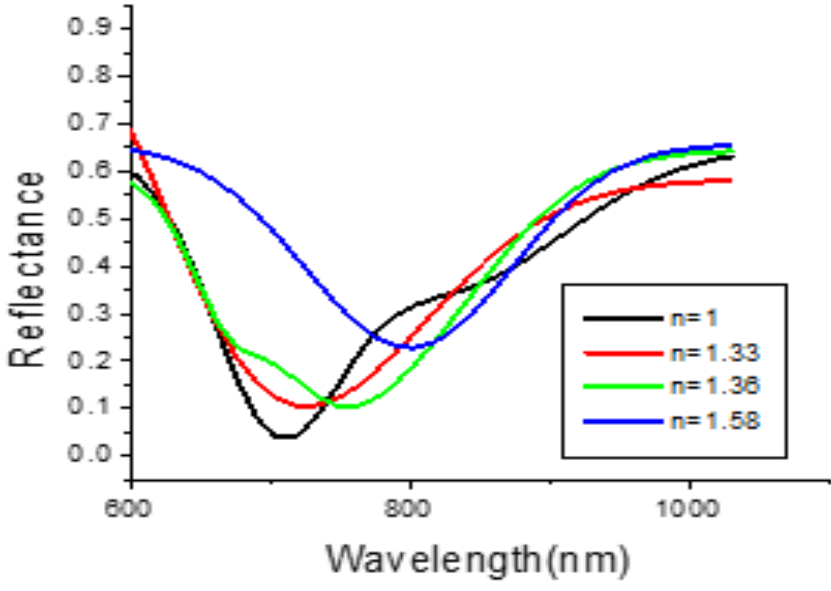

(b)

Figure 7. SPR curves of the optical fiber sensor

From the fitting results, the resonance wavelength of SPR sensor change with the change refractive index as shown in Figure 8.

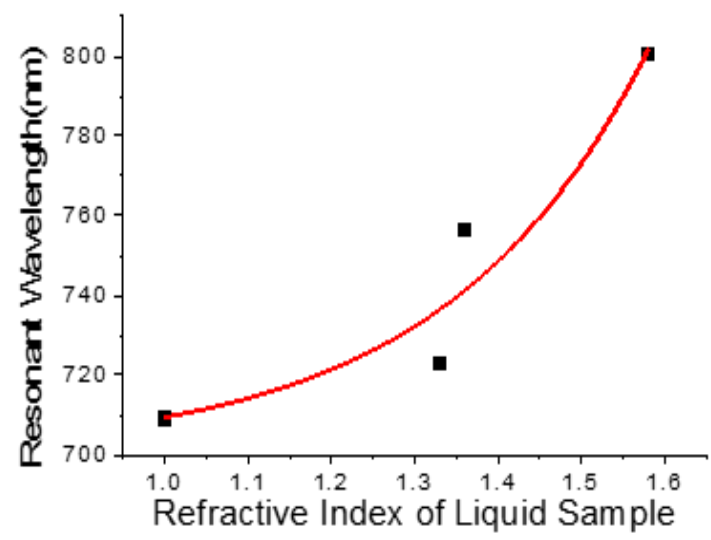

Figure 8. Fitting result of fiber SPR sensor in refractive index measurement

The sensitivity of the refractive index plasmonic sensor obtained by fitting depends on the total sensing range from 1 to 1.58 is about $298 \mathrm{~nm} / \mathrm{RIU}$ according to the relationship $\frac{\Delta \lambda_{\text {res }}}{\Delta n}$, where $\Delta n$ is the variation of refractive index of solvents and $\Delta \lambda_{\text {res }}$ is the variation of resonance wavelength. The sensitivity (S), resolution (R), the signal to noise ratio (SNR) and the figure of merit (FOM), were the highest with increase refractive index of the medium as shown in Table 2.

Table 2. Performance parameters for MMF- SPR sensor obtained for different environmental refractive indices

\begin{tabular}{cccc}
$\mathbf{S}(\mathbf{n m} / \mathbf{R I U})$ & $\mathbf{F O M}$ & $\mathbf{S N R}$ & $\mathbf{R} \times \mathbf{1 0}^{\mathbf{- 4}}$ \\
\hline 298 & 2.316 & 0.147 & 4.31
\end{tabular}




\section{Conclusion}

In this work, a simple optical fiber sensor based on SPR was prepared and employed for testing refractive index changes of several solvents. Satisfy the SPR phenomena depend on different core diameter of optical fiber instead of the etching method for cladding as traditional fibers to leakage light into cladding to excite surface plasmon. $\mathrm{A}_{\mathrm{u}}$ coated as SPR metal film, the experiment results explained the response of this sensor is resonance wavelength dip position shifts linearly to longer wavelengths as the refractive index increase. The ranging from the spectrum that occurs surface plasmon resonance start from visible to NIR. The SPR sensor that fabricated can be used as a refractometer sensor the sensitivity of the sensor reach to $298 \mathrm{~nm} / \mathrm{RIU}$.

\section{References}

[1] E. Klantsataya, P. Jia, H. Ebendorff-Heidepriem, T. M. Monro, and A. François, "Plasmonic fiber optic refractometric sensors: From conventional architectures to recent design trends," Sensors, vol. 17, no. 1, p. 12, 2017.

[2] J. Yan et al., "Improving the sensitivity of fiber-optic SPR sensor via radially polarized beam excitation," Chinese Opt. Lett., vol. 7, no. 10, pp. 909-911, 2009.

[3] C. Ronot-Trioli, A. Trouillet, C. Veillas, and H. Gagnaire, "Monochromatic excitation of surface plasmon resonance in an optical-fibre refractive-index sensor," Sensors Actuators A Phys., vol. 54, no. 1-3, pp. 589-593, 1996.

[4] Y. Huang, D. Wu, C.-J. Chuang, B. Nie, H. Cui, and W. Yun, "Theoretical analysis of tapered fiber optic surface plasmon resonance sensor for voltage sensitivity," Opt. Fiber Technol., vol. 22, pp. 42-45, 2015.

[5] Y. Zhang, T. Zhou, B. Han, A. Zhang, and Y. Zhao, "Optical bio-chemical sensors based on whispering gallery mode resonators," Nanoscale, vol. 10, no. 29, pp. 13832-13856, 2018.

[6] Z. Chen, K. Han, and Y.-N. Zhang, "Reflective Fiber Surface Plasmon Resonance Sensor for High-Sensitive Mercury Ion Detection," Appl. Sci., vol. 9, no. 7, p. 1480, 2019.

[7] A. K. Sharma, R. Jha, and B. D. Gupta, "Fiber-optic sensors based on surface plasmon resonance: a comprehensive review," IEEE Sens. J., vol. 7, no. 8, pp. 1118-1129, 2007.

[8] W. L. Barnes, A. Dereux, and T. W. Ebbesen, "Surface plasmon subwavelength optics," Nature, vol. 424, no. 6950, p. 824, 2003.

[9] R. P. H. Kooyman, "Physics of surface plasmon resonance," Handb. Surf. Plasmon Reson., vol. 1, 2008.

[10] X. Liu, D. Song, Q. Zhang, Y. Tian, L. Ding, and H. Zhang, "Wavelength-modulation surface plasmon resonance sensor," TrAC Trends Anal. Chem., vol. 24, no. 10, pp. 887-893, 2005.

[11] J. Homola et al., "Optical biosensing using surface plasmon resonance spectroscopy," in Chemical, Biochemical and Environmental Fiber Sensors IX, 1997, vol. 3105, pp. 318-324.

[12] N. Cennamo, D. Massarotti, L. Conte, and L. Zeni, "Low cost sensors based on SPR in a plastic optical fiber for biosensor implementation," Sensors, vol. 11, no. 12, pp. 11752 11760, 2011. 